\title{
edoc
}

Institutional Repository of the University of Basel

University Library

Schoenbeinstrasse 18-20

CH-4056 Basel, Switzerland

http://edoc.unibas.ch/

\section{Coordinated balancing of muscle oxidative metabolism through PGC- $1 \alpha$ increases metabolic flexibility and preserves insulin sensitivity}

\author{
Summermatter, S. and Troxler, H. and Santos, G. and Handschin, C.
}

Posted at edoc, University of Basel

Official URL: http://edoc.unibas.ch/dok/A6001473

Originally published as:

Summermatter, S. and Troxler, H. and Santos, G. and Handschin, C.. (2011) Coordinated balancing of muscle oxidative metabolism through PGC-1 $\alpha$ increases metabolic flexibility and preserves insulin sensitivity. Biochemical and biophysical research communications, Vol. 408, H. 1. S. 180-185. 
Coordinated balancing of muscle oxidative metabolism through PGC-1 $\alpha$ increases metabolic flexibility and preserves insulin sensitivity

\author{
Serge Summermatter ${ }^{1}$, Heinz Troxler ${ }^{2}$, Gesa Santos ${ }^{1}$, Christoph Handschin ${ }^{1}$
}

${ }^{1}$ Biozentrum, Division of Pharmacology/Neurobiology, University of Basel, Klingelbergstrasse 50-70, CH-4056 Basel, Switzerland

${ }^{2}$ Division of Clinical Chemistry and Biochemistry, Department of Pediatrics, University Children's Hospital, University of Zurich, Steinwiesstrasse 75, CH-8032 Zurich, Switzerland

Published in Biochem Biophys Res Commun. 2011 Apr 29;408(1):180-5. PMID: 21501593. doi: 10.1016/j.bbrc.2011.04.012

Copyright $\subseteq$ Elsevier; Biochemical and Biophysical Research Communications 


\title{
Coordinated balancing of muscle oxidative metabolism through PGC-1 $\alpha$ increases metabolic flexibility and preserves insulin sensitivity
}

\author{
Serge Summermatter ${ }^{1}$, Heinz Troxler $^{2}$, Gesa Santos ${ }^{1}$, Christoph Handschin ${ }^{1}$ \\ ${ }^{1}$ Biozentrum, Division of Pharmacology/Neurobiology, University of Basel, Klingelbergstrasse 50-70, CH-4056 \\ Basel, Switzerland \\ ${ }^{2}$ Division of Clinical Chemistry and Biochemistry, Department of Pediatrics, University Children's Hospital, \\ University of Zurich, Steinwiesstrasse 75, CH-8032 Zurich, Switzerland
}

\author{
Corresponding author: \\ Dr. Christoph Handschin \\ Biozentrum, Div. of Pharmacology/Neurobiology \\ University of Basel \\ Klingelbergstrasse 50-70 \\ CH-4056 Basel \\ SWITZERLAND \\ Phone: +41612672378 \\ Fax: +41 612672208 \\ Email: christoph.handschin@unibas.ch
}

\begin{abstract}
The peroxisome proliferator-activated receptor $\gamma$ coactivator $1 \alpha(\mathrm{PGC}-1 \alpha)$ enhances oxidative metabolism in skeletal muscle. Excessive lipid oxidation and electron transport chain activity can, however, lead to the accumulation of harmful metabolites and impair glucose homeostasis. Here, we investigated the effect of over-expression of PGC-1 $\alpha$ on metabolic control and generation of insulin desensitizing agents in extensor digitorum longus (EDL), a muscle that exhibits low levels of PGC-1 $\alpha$ in the untrained state and minimally relies on oxidative metabolism. We demonstrate that PGC-1 $\alpha$ induces a strictly balanced substrate oxidation in EDL by concomitantly promoting the transcription of activators and inhibitors of lipid oxidation. Moreover, we show that PGC-1 $\alpha$ enhances the potential to uncouple oxidative phosphorylation. Thereby, PGC-1 $\alpha$ boosts elevated, yet tightly regulated oxidative metabolism devoid of side products that are detrimental for glucose homeostasis.

Accordingly, PI3K activity, an early phase marker for insulin resistance, is preserved in EDL muscle. Our findings suggest that PGC- $1 \alpha$ coordinately coactivates the simultaneous transcription of gene clusters implicated in the positive and negative regulation of oxidative metabolism and thereby increases metabolic flexibility. Thus, in mice fed a normal chow diet, over-expression of PGC- $1 \alpha$ does not alter insulin sensitivity and the metabolic adaptations elicited by PGC-1 $\alpha$ mimic the beneficial effects of endurance training on muscle metabolism in this context.
\end{abstract}

Key words: metabolic flexibility, exercise mimetics, insulin resistance, glucose homeostasis, PGC-1 $\alpha$

\section{Highlights}

$>$ PGC-1 $\alpha$ enhances muscle oxidative capacity $>$ PGC- $1 \alpha$ promotes concomitantly positive and negative regulators of lipid oxidation $>$ Regulator abundance enhances metabolic flexibility 
and balances oxidative metabolism $>$ Balanced oxidation prevents detrimental acylcarnitine and ROS generation>Absence of detrimental metabolites preserves insulin sensitivity 


\section{Introduction}

The increasing prevalence of metabolic disorders in our modern society represents a serious medical and economic challenge for the future [1]. A sedentary lifestyle and a low metabolic efficiency substantially contribute to the development of such diseases [1;2]. Inversely, regular exercise ameliorates or partially prevents these conditions.

The peroxisome proliferator-activated receptor $\gamma$ coactivator $1 \alpha$ largely mediates the adaptive effects of exercise in skeletal muscle $[3 ; 4 ; 5]$. PGC- $1 \alpha$ is a transcriptional coactivator and is known to promote the expression of several transcription factors, which it subsequently coactivates [6]. An endurance trained status can be pheno-copied by muscle-specific overexpression of PGC-1 $\alpha$, even in the absence of physical activity [7; $8 ; 9]$. PGC-1 $\alpha$ induces mitochondrial biogenesis, improves oxygen supply to muscle by promoting angiogenesis [10], increases peak oxygen consumption, and endurance capacity [8]. Moreover, PGC-1 $\alpha$ drives fiber-type switching from fast, glycolytic towards slow, oxidative fibers [7]. Most importantly, PGC-1 $\alpha$ promotes lipid and glucose provision and enhances oxidative metabolism [3; 11].

A reduction in metabolic efficiency is associated with insulin resistance in rodents and humans [12]. Regular physical activity is thus believed to exert a beneficial effect by elevating the metabolic capacity [13]. However, a careful regulation of this process seems important since excessive or dysbalanced oxidative metabolism impairs insulin sensitivity [14]. For example, acylcarnitines are generated when the amount of lipids fluxed into $\beta-$ oxidation exceeds the capacity of the Krebs cycle and/or oxidative phosphorylation (OXPHOS) [14]. These detrimental lipid species are subsequently released into the circulation and contribute to the development of insulin resistance. Similarly, the proton gradient that generates the mitochondrial membrane potential is a potential source of reactive oxygen species (ROS), which are also implicated in the etiology of insulin resistance [15]. Currently, it is unresolved whether the induction of oxidative metabolism through PGC-1 $\alpha$ leads to generation of such metabolites in skeletal muscle in mice fed a normal chow diet.

We now investigated the impact of elevated oxidative metabolism driven by PGC-1 $\alpha$ on the production of acylcarnitines and/or ROS in the extensor digitorum longus (EDL) muscle, on whole body glucose homeostasis and on PI3K activity as an early marker of skeletal muscle insulin sensitivity. Finally, we describe the molecular mechanisms that fine-tune oxidative metabolism and increase metabolic flexibility upon ectopic expression of PGC- $1 \alpha$.

\section{Materials and Methods}

\section{Animals}

Muscle-specific PGC-1 $\alpha$ transgenic mice (MPGC-1 $\alpha$ TG) [7] and control littermates were maintained in a conventional facility with a fixed 12-h light/dark cycle on a commercial pellet chow diet and free access to tap water. Studies were performed according to criteria outlined for the care and use of laboratory animals and with approval of the Swiss authorities.

\section{RNA extraction and $R T-P C R$}

Frozen tissues were homogenized under liquid nitrogen and total RNA was isolated using Trizol reagent (Invitrogen). RNA concentrations were adjusted and reverse transcription was carried out using random hexamer primers (Promega). Real-time PCR analysis (Power SYBR 
Green Master Mix, Applied Biosystems) was performed using the ABI Prism 7000 Sequence Detector. Relative expression levels for each gene of interest were calculated with the $\Delta \Delta \mathrm{Ct}$ method and normalized to the expression of the Tata box binding protein (TBP).

Mitochondrial DNA content was quantified by determining the ratio of mitochondrial cytochrome c oxidase 2 (Cox2) to nuclear intron of $\beta$-globin by RT-PCR of isolated DNA.

\section{Lipid oxidation}

Palmitate oxidation was measured as described by Dyck et al [16]. Radioactivity in the water phase was quantified using a Beckman liquid scintillation counter.

All values were corrected for initial tissue weight and expressed as fold change compared to control animals.

\section{Muscle ROS}

To monitor intracellular generation of ROS, $2^{\prime}$, $7^{\prime}$-dichlorofluorescein diacetate $\left(\mathrm{H}_{2} \mathrm{DCFDA}\right)$ (Sigma) was used. EDL muscles were harvested in cold-buffered medium ( 5 mmol/1 HEPES in PBS) and immediately frozen in liquid nitrogen. After rapid thawing, medium was discarded. Samples were exposed to $8 \mu \mathrm{mol} / 1 \mathrm{H}_{2}$ DCFDA dissolved in $400 \mu \mathrm{l}$ fresh medium and were incubated at $37^{\circ} \mathrm{C}$ for $30 \mathrm{~min}$ under agitation. Medium was then removed, samples were homogenized and further incubated in lysis buffer (0.1\% SDS, Tris-HCl, $\mathrm{pH} 7.4)$. Samples were centrifuged at 14'000rpm. Supernatants were collected and subjected to fluorescence analysis at $520 \mathrm{~nm}$ under excitation at $485 \mathrm{~nm}$ using a microplate reader. Dichlorofluorescein fluorescence corresponds to the ROS production generated during the ex vivo sample incubation.

\section{Acylcarnitine determination}

Acylcarnitines were extracted from dried blood spots using methanol containing eight isotopically labeled internal standards (Cambridge Isotopes Laboratories) and analyzed without prior sample derivatization. Precursor ions of $\mathrm{m} / \mathrm{z} 85$ in the mass range of $\mathrm{m} / \mathrm{z} 150$ to 450 are acquired on a PerkinElmer API 365 LC-ESI-MS/MS instrument.

\section{Glucose and insulin tolerance tests}

Animals were fasted for $16 \mathrm{~h}$ and $6 \mathrm{~h}$ before i.p. injection of $2 \mathrm{~g} / \mathrm{kg}$ glucose and $0.8 \mathrm{U} / \mathrm{kg}$ insulin, respectively. Blood was obtained at intervals of 15 minutes from the tail vein, and glucose levels were determined using a standard glucometer.

\section{PI3K activity}

Muscles were dissected, immediately frozen in liquid nitrogen and homogenized under liquid nitrogen. IRS-1 associated PI3K activity was assessed as previously described $[17 ; 18]$.

\section{Mitochondrial mass and membrane potential}

Myoblasts isolated from MPGC-1 $\alpha$ TG mice and control littermates were permitted to fuse into multinucleated myotubes. Mitochondrial mass and membrane potential were measured in myotubes using Mitotracker Green (MTG; Molecular Probes) and Tetramethylrhodamine ethyl ester (TMRE; Molecular Probes). In brief, cells were incubated with differentiation media containing 100nM MTG or $100 \mathrm{nM}$ TMRE for $20 \mathrm{~min}$ at $37^{\circ} \mathrm{C}$ in a humidified atmosphere of $5 \% \mathrm{CO}_{2}$. After $20 \mathrm{~min}$, cells were washed three times with PBS, and a microplate reader was used to measure MTG or TMRE fluorescence (excitation 490 or 549 $\mathrm{nm}$, emission 516 or $574 \mathrm{~nm}$, respectively). Fluorescent values for the mitochondrial membrane potential were corrected for mitochondrial mass.

\section{Data analysis and statistics}


All data are presented as means \pm SE. The data were analyzed by 2-tailed, unpaired Student's $t$ test or Mann-Whitney test when the difference between the two SDs was significantly different. Levels of significance are indicated as follows: ${ }^{* * *} \mathrm{p}<0.001,{ }^{*} \mathrm{p}<0.01,{ }^{*} \mathrm{p}<0.05$.

\section{Results}

\section{Increased mitochondrial biogenesis and elevated mitochondrial DNA content in EDL muscle of MPGC-1a TG mice}

PGC-1 $\alpha$ gene expression in EDL muscle is $\sim 9$ times higher in MPGC- $1 \alpha$ TG mice than in controls $(\mathrm{P}<0.001)$ and no potentially compensatory changes in the expression of PGC-1 $1 \beta$ or PGC-1-related coactivator are observed (Figure 1A). Consequently, mRNA levels of the mitochondrial regulators estrogen-related receptor $\alpha$ and mitochondrial transcription factor $\mathrm{A}$ as well as the mitochondrial proteins NADH-ubiquinone oxidoreductase Fe-S protein 1, $\mathrm{NADH}$-ubiquinone oxidoreductase flavoprotein 2, cytochrome $\mathrm{c}$ and medium chain acyl-coA dehydrogenase are significantly increased in transgenic animals (Figure 1B). Furthermore, MPGC-1 $\alpha$ TG mice display elevated mitochondrial DNA content $(+64 \%(\mathrm{P}<0.001))$ (Figure 1C) and increased mitochondrial mass (Figure 1D).

\section{Enhanced lipid oxidation is in balance with the Krebs cycle in MPGC-1a TG mice}

We next examined whether these mice subsequently show altered mitochondrial lipid metabolism in EDL. Expression of genes involved in the activation of fatty acid $\beta$-oxidation $(\mathrm{CPT}-1 \mathrm{~b}$ and MCD) are up-regulated in MPGC- $1 \alpha$ TG mice $(+237 \%$ and $+169 \%$ respectively); reaching statistical significance only for $\mathrm{CPT}-1 \mathrm{~b}(\mathrm{P}<0.01)$ (Figure $2 \mathrm{~A})$. Surprisingly, ACC2, which is implicated in the inhibition of $\beta$-oxidation, is similarly induced $(+189 \%, \mathrm{P}<0.01)$ (Figure 2A). Nevertheless, the overall $\beta$-oxidation rate is increased in MPGC- $1 \alpha$ TG mice by $+62 \%(\mathrm{P}<0.05)$ (Figure $2 \mathrm{~B})$. To evaluate downstream events of lipid oxidation, we determined mRNA expression of citrate synthase. Our results show that mRNA levels of citrate synthase are significantly higher in MPGC- $1 \alpha$ TG mice than in controls $(+299 \%, \mathrm{P}<0.001)$ (Figure 2C).

Excessive or dysbalanced lipid oxidation contributes to skeletal muscle insulin resistance by increasing acylcarnitines production and secretion [14]. We thus tested whether the elevated lipid oxidation in MPGC-1 $\alpha$ TG mice is associated with the production of such detrimental metabolites by LC-ESI-MS/MS. This technique is very sensitive and even enables the detection of mild defects in lipid oxidation. No significant changes between MPGC-1 $\alpha$ TG and control animals were found for any of these carnitine esters in the circulation (Figure 2D).

Elevated oxidative phosphorylation, but unchanged ROS generation in MPGC-1a TG mice Increased fatty acid oxidation and Krebs cycle activity ultimately feed into the mitochondrial oxidative phosphorylation (OXPHOS) for ATP production. Accordingly, genes encoding different subunits of the OXPHOS system are elevated in MPGC-1 $\alpha$ TG animals (Figure 3A). Interestingly, expression of the uncoupling protein 3 (UCP3) is likewise increased (Figure $3 \mathrm{~A})$. UCP3 might dissipate the proton gradient in the mitochondria and thereby reduce the membrane potential and ATP synthesis. However, the net effect of these seemingly counteracting processes is an increase in membrane potential by $+4 \%(\mathrm{P}<0.05)$ (Figure $3 \mathrm{~B})$.

To study the impact of an elevated membrane potential on ROS production, we evaluated mRNA expression of genes implicated in ROS production and detoxification. Mitochondrialencoded superoxide dismutase 2 (SOD2) is increased in MPGC- $1 \alpha$ TG mice $(+127 \%$, $\mathrm{P}<0.01)$, whereas nuclear-encoded SOD1 is decreased by $-27 \%(\mathrm{P}<0.05)$ (Figure $3 \mathrm{C})$. Furthermore, the expression of genes involved in $\mathrm{H}_{2} \mathrm{O}_{2}$ detoxification, namely catalase, 
glutathione reductase and glutathione peroxidase, does not differ between MPGC-1 $\alpha$ TG and control mice (Figure 3C). As a consequence, overall $\mathrm{H}_{2} \mathrm{O}_{2}$ production is unaltered in MPGC$1 \alpha$ TG mice suggesting a tightly regulated balance between OXPHOS and ROS metabolism (Figure 3D).

\section{Normal glucose homeostasis despite enhanced muscle oxidative metabolism in MPGC-1a TG mice}

The ability of MPGC-1 $\alpha$ TG and control mice to deal with an acute glucose load was assessed by a glucose tolerance test. Both genotypes show similar glucose excursion curves (Figure 4A). Insulin tolerance tests were used to determine whether systemic insulin sensitivity is altered in MPGC-1 $\alpha$ TG mice. The capacities of MPGC-1 $\alpha$ TG and control mice to regulate blood glucose levels after a bolus of insulin are indistinguishable (Figure 4B). Finally, IRS-1 associated PI3K activity, which is a very early marker for defects in glucose homeostasis, does not differ significantly between the two genotypes (Figure 4C).

\section{Discussion}

The increasing prevalence of metabolic disorders poses a major public health problem [1] and a sedentary lifestyle strongly promotes the development of such metabolic diseases [2]. Although most people are aware of the necessity of adequate physical activity, many are unable or unwilling to achieve the level that is required to elicit health benefits. The development of drugs that mimic the plastic changes elicited by exercise thus constitutes an intriguing pharmacological approach to this problem $[13 ; 19]$. PGC-1 $\alpha$ is an attractive target for such interventions because this protein is central to endurance exercise adaptation and by itself is sufficient to induce a trained phenotype along with an elevated metabolic capacity [3; $4 ; 5 ; 13]$. Moreover, known experimental "exercise mimetics", including activators for AMPdependent protein kinase, at least in part act through PGC-1 $\alpha$ [20]. Importantly however, when oxidative metabolism is promoted in excess, or when only individual oxidative pathways are increased without simultaneous up-regulation of subsequent steps, adverse effects on metabolic functions occur [14; 15].

We now show that PGC-1 $\alpha$ coordinately induces genes that promote lipid oxidation (CPT-1b and MCD), Krebs cycle (citrate synthase) and OXPHOS (subunits of complexes I-V) and thereby couples all major oxidative steps in EDL muscle. Intriguingly, we found that PGC-1 $\alpha$ not only controls the expression of positive, but also of negative regulators of fatty acid oxidation and OXPHOS. Thus, the levels of ACC2, an inhibitior of lipid oxidation, and of UCP3, which uncouples mitochondria and thereby reduces OXPHOS, are both elevated in the transgenic animals. Importantly, while the overall gene expression pattern is ambiguous, the net rates are nonetheless clearly showing an increased oxidation of fatty acids and an elevated mitochondrial membrane potential. Therefore, our findings imply that the increased $\beta$ oxidation and the subsequent Krebs cycle and OXPHOS are tightly regulated and balanced by PGC-1 $\alpha$ in EDL muscle (Figure 4D). The concomitant induction of both positive and negative regulators of fatty acid oxidation and OXPHOS through PGC- $1 \alpha$ boosts metabolic flexibility and restrains excessive oxidation.

As a consequence of the complex gene expression controlled by PGC-1 $\alpha$, detrimental side products of $\beta$-oxidation or increased ROS, which both have been linked to the etiology of insulin resistance $[14 ; 15]$, are not observed in our animal model with ectopically expressed PGC-1 $\alpha$ in muscle despite significantly higher oxidative activity. A relationship between acylcarnitines and insulin resistance has recently been established in rodents and type 2 
diabetic patients $[14 ; 21 ; 22]$. Elevated acylcarnitines were reported in blood and skeletal muscle from insulin-resistant rats, but there was no increase observed in the liver, suggesting that blood acylcarnitines mainly originated from muscle [14]. In the human study, long, shortand medium-chain acylcarnitines increased in diabetic patients compared to lean individuals [21]. The accumulation of short-chain acylcarnitines, which represent late degradation products of lipid catabolism, is suggestive of inefficient fatty acid oxidation or poor coupling between lipid oxidation and OXPHOS in diabetic subjects [21]. The acylcarnitine profile in our study does not reveal any chain-length dependent alterations between wild-type and transgenic animals. We even observe a trend towards lower levels of short-chain acylcarnitines. This is consistent with an improved oxidative capacity and tight coupling of $\beta$ oxidation, Krebs cycle and OXPHOS. Even when further subdivided into different lipid classes, such as saturated, mono- or polyunsaturated acylcarnitines, no differences between control and transgenic animals are detectable. This detailed inspection might be important, as analogous to fatty acids, saturated species of acylcarnitines might exert a more detrimental effect on insulin sensitivity than mono- or polyunsaturated species. The exact connection between acylcarnitines and insulin sensitivity is a future issue that needs to be addressed. A possible mechanism might involve the induction of $\mathrm{NF \kappa B}$ through acycarnitines as previously demonstrated in vitro [23].

Finally, while an effect of PGC-1 $\alpha$ on ROS detoxification and mitochondrial uncoupling has been shown in previous studies in vitro [24;25], we now for the first time demonstrate unchanged levels of ROS in muscle of PGC-1 $\alpha$ transgenic mice compared to wild-type controls. These findings suggest a tight balance between mitochondrial activity, a major source of ROS, and ROS detoxification in muscle with elevated PGC-1 $\alpha$. This balance might be achieved by limited production of ROS via UCP3-mediated mitochondrial uncoupling in combination with enhanced ROS detoxification, e.g. through elevated SOD2.

Although we focused our studies on EDL muscle, PGC-1 $\alpha$ is obviously elevated in all skeletal muscles in our experimental mouse model. Interestingly, the enhancement of muscle oxidative metabolism in skeletal muscle does not influence whole body glucose homoeostasis as glucose and insulin tolerance remain normal in the transgenic mice fed a normal chow diet. The possibility arises, however, that alterations in insulin sensitivity occur in skeletal muscle, but that compensatory mechanisms in other tissues restore whole body glucose homeostasis. Thus, to directly determine insulin sensitivity in skeletal muscle, PI3K, a key molecule in insulin signaling and an early marker for insulin resistance, was investigated as PI3K activity is already impaired in the pre-diabetic state [17]. We demonstrate that PI3K activity in EDL muscle is similar in wild type and transgenic animals, corroborating further that peripheral insulin sensitivity in skeletal muscle is preserved. Intriguingly, sedentary PGC- $1 \alpha$ musclespecific transgenic mice on a high fat diet develop insulin resistance even faster than wildtype control mice [26]. We have previously demonstrated that as part of the exercise adaptations controlled by PGC-1 $\alpha$, these mice have higher de-novo lipogenesis rates and thus exhibit an accumulation of intramyocellular lipids [11]. These data suggested that the use of putative exercise mimetics in sedentary individuals on a Western diet could even exacerbate pathological conditions. Our findings reported here show that without excess dietary lipids, ectopically expressed PGC- $1 \alpha$ mediates beneficial adaptations in skeletal muscle, even in sedentary mice.

Taken together, our findings provide new insights into the molecular mechanisms by which PGC- $1 \alpha$ coordinates oxidative metabolism. By tightly balancing lipid oxidation and mitochondrial electron transport, elevated muscle PGC- $1 \alpha$ avoids the generation of detrimental side products that impair insulin sensitivity in mice fed a regular chow diet. Our findings imply that while induction of PGC-1 $\alpha$ per se does not improve insulin sensitivity in 
this experimental context, this transcriptional coactivator increases the metabolic flexibility of skeletal muscle.

\section{Acknowledgements}

We thank Urs A. Meyer (Biozentrum, Basel) and our colleagues in the laboratory for discussions and comments on the manuscript. This project was funded by the Swiss National Science Foundation, the Muscular Dystrophy Association USA (MDA), the SwissLife 'Jubiläumsstiftung für Volksgesundheit und medizinische Forschung', the Swiss Society for Research on Muscle Diseases (SSEM), the Swiss Diabetes Association, the Roche Research Foundation, the United Mitochondrial Disease Foundation (UMDF), the Association Française contre les Myopathies (AFM), the Gebert-Rüf Stiftung (GRS) and the University of Basel.

Conflict of interest: The authors have no conflict of interest.

\section{References}

[1] S. Crunkhorn, and M.E. Patti, Links between thyroid hormone action, oxidative metabolism, and diabetes risk? Thyroid 18 (2008) 227-37.

[2] F.W. Booth, M.J. Laye, S.J. Lees, R.S. Rector, and J.P. Thyfault, Reduced physical activity and risk of chronic disease: the biology behind the consequences. Eur J Appl Physiol 102 (2008) 381-90.

[3] C. Handschin, and B.M. Spiegelman, Peroxisome proliferator-activated receptor gamma coactivator 1 coactivators, energy homeostasis, and metabolism. Endocr Rev 27 (2006) 728-35.

[4] J. Lin, C. Handschin, and B.M. Spiegelman, Metabolic control through the PGC-1 family of transcription coactivators. Cell Metab 1 (2005) 361-70.

[5] C. Handschin, and B.M. Spiegelman, The role of exercise and PGC1alpha in inflammation and chronic disease. Nature 454 (2008) 463-9.

[6] B.N. Finck, and D.P. Kelly, PGC-1 coactivators: inducible regulators of energy metabolism in health and disease. J Clin Invest 116 (2006) 615-22.

[7] J. Lin, H. Wu, P.T. Tarr, C.Y. Zhang, Z. Wu, O. Boss, L.F. Michael, P. Puigserver, E. Isotani, E.N. Olson, B.B. Lowell, R. Bassel-Duby, and B.M. Spiegelman, Transcriptional co-activator PGC-1 alpha drives the formation of slow-twitch muscle fibres. Nature 418 (2002) 797-801.

[8] J.A. Calvo, T.G. Daniels, X. Wang, A. Paul, J. Lin, B.M. Spiegelman, S.C. Stevenson, and S.M. Rangwala, Muscle-specific expression of PPARgamma coactivator-1alpha improves exercise performance and increases peak oxygen uptake. J Appl Physiol 104 (2008) 1304-12.

[9] M. Sandri, J. Lin, C. Handschin, W. Yang, Z.P. Arany, S.H. Lecker, A.L. Goldberg, and B.M. Spiegelman, PGC-1alpha protects skeletal muscle from atrophy by suppressing FoxO3 action and atrophy-specific gene transcription. Proc Natl Acad Sci U S A 103 (2006) 16260-5.

[10] Z. Arany, S.Y. Foo, Y. Ma, J.L. Ruas, A. Bommi-Reddy, G. Girnun, M. Cooper, D. Laznik, J. Chinsomboon, S.M. Rangwala, K.H. Baek, A. Rosenzweig, and B.M. Spiegelman, HIF-independent regulation of VEGF and angiogenesis by the transcriptional coactivator PGC-1alpha. Nature 451 (2008) 1008-12.

[11] S. Summermatter, O. Baum, G. Santos, H. Hoppeler, and C. Handschin, Peroxisome proliferator-activated receptor $\{$ gamma $\}$ coactivator $1\{$ alpha\} (PGC-1\{alpha\}) 
promotes skeletal muscle lipid refueling in vivo by activating de novo lipogenesis and the pentose phosphate pathway. J Biol Chem 285 (2010) 32793-800.

[12] A.E. Civitarese, and E. Ravussin, Mitochondrial energetics and insulin resistance. Endocrinology 149 (2008) 950-4.

[13] A.P. Russell, PGC-1alpha and exercise: important partners in combating insulin resistance. Curr Diabetes Rev 1 (2005) 175-81.

[14] T.R. Koves, J.R. Ussher, R.C. Noland, D. Slentz, M. Mosedale, O. Ilkayeva, J. Bain, R. Stevens, J.R. Dyck, C.B. Newgard, G.D. Lopaschuk, and D.M. Muoio, Mitochondrial overload and incomplete fatty acid oxidation contribute to skeletal muscle insulin resistance. Cell Metab 7 (2008) 45-56.

[15] N. Houstis, E.D. Rosen, and E.S. Lander, Reactive oxygen species have a causal role in multiple forms of insulin resistance. Nature 440 (2006) 944-8.

[16] D.J. Dyck, S.J. Peters, J. Glatz, J. Gorski, H. Keizer, B. Kiens, S. Liu, E.A. Richter, L.L. Spriet, G.J. van der Vusse, and A. Bonen, Functional differences in lipid metabolism in resting skeletal muscle of various fiber types. Am J Physiol 272 (1997) E340-51.

[17] S. Summermatter, D. Mainieri, A.P. Russell, J. Seydoux, J.P. Montani, A. Buchala, G. Solinas, and A.G. Dulloo, Thrifty metabolism that favors fat storage after caloric restriction: a role for skeletal muscle phosphatidylinositol-3-kinase activity and AMPactivated protein kinase. Faseb J 22 (2008) 774-85.

[18] S. Summermatter, H. Marcelino, D. Arsenijevic, A. Buchala, O. Aprikian, F. Assimacopoulos-Jeannet, J. Seydoux, J.P. Montani, G. Solinas, and A.G. Dulloo, Adipose tissue plasticity during catch-up fat driven by thrifty metabolism: relevance for muscle-adipose glucose redistribution during catch-up growth. Diabetes 58 (2009) 2228-37.

[19] V.A. Narkar, M. Downes, R.T. Yu, E. Embler, Y.X. Wang, E. Banayo, M.M. Mihaylova, M.C. Nelson, Y. Zou, H. Juguilon, H. Kang, R.J. Shaw, and R.M. Evans, AMPK and PPARdelta agonists are exercise mimetics. Cell 134 (2008) 405-15.

[20] A.L. Carey, and B.A. Kingwell, Novel pharmacological approaches to combat obesity and insulin resistance: targeting skeletal muscle with 'exercise mimetics'. Diabetologia 52 (2009) 2015-26.

[21] S.J. Mihalik, B.H. Goodpaster, D.E. Kelley, D.H. Chace, J. Vockley, F.G. Toledo, and J.P. DeLany, Increased levels of plasma acylcarnitines in obesity and type 2 diabetes and identification of a marker of glucolipotoxicity. Obesity (Silver Spring) 18 (2010) 1695-700.

[22] J. An, D.M. Muoio, M. Shiota, Y. Fujimoto, G.W. Cline, G.I. Shulman, T.R. Koves, R. Stevens, D. Millington, and C.B. Newgard, Hepatic expression of malonyl-CoA decarboxylase reverses muscle, liver and whole-animal insulin resistance. Nat Med 10 (2004) 268-74.

[23] S.H. Adams, C.L. Hoppel, K.H. Lok, L. Zhao, S.W. Wong, P.E. Minkler, D.H. Hwang, J.W. Newman, and W.T. Garvey, Plasma acylcarnitine profiles suggest incomplete long-chain fatty acid beta-oxidation and altered tricarboxylic acid cycle activity in type 2 diabetic African-American women. J Nutr 139 (2009) 1073-81.

[24] J. St-Pierre, J. Lin, S. Krauss, P.T. Tarr, R. Yang, C.B. Newgard, and B.M. Spiegelman, Bioenergetic analysis of peroxisome proliferator-activated receptor gamma coactivators 1alpha and 1beta (PGC-1alpha and PGC-1beta) in muscle cells. J Biol Chem 278 (2003) 26597-603.

[25] J. St-Pierre, S. Drori, M. Uldry, J.M. Silvaggi, J. Rhee, S. Jager, C. Handschin, K. Zheng, J. Lin, W. Yang, D.K. Simon, R. Bachoo, and B.M. Spiegelman, Suppression of reactive oxygen species and neurodegeneration by the PGC-1 transcriptional coactivators. Cell 127 (2006) 397-408. 
[26] C.S. Choi, D.E. Befroy, R. Codella, S. Kim, R.M. Reznick, Y.J. Hwang, Z.X. Liu, H.Y. Lee, A. Distefano, V.T. Samuel, D. Zhang, G.W. Cline, C. Handschin, J. Lin, K.F. Petersen, B.M. Spiegelman, and G.I. Shulman, Paradoxical effects of increased expression of PGC-1alpha on muscle mitochondrial function and insulin-stimulated muscle glucose metabolism. Proc Natl Acad Sci U S A 105 (2008) 19926-31.

\section{Figure Legends}

Figure 1. Effect of PGC-1 $\alpha$ on mitochondrial gene expression and biogenesis in EDL. (A and B) Relative gene expression of PGC-1 $\alpha$, PGC-1 $\beta$ and PRC (A) and mitochondrial target genes (B) was measured by RT-PCR and expressed as fold change over controls. Abbreviations: PGC-1, peroxisome proliferator-activated receptor $\gamma$ coactivator 1; PRC, PGC1-related coactivator; ERR $\alpha$, estrogen-related receptor $\alpha$; TFAM, mitochondrial transcription factor A; Ndufs1, NADH-ubiquinone oxidoreductase Fe-S protein 1; Ndufv2, NADHubiquinone oxidoreductase flavoprotein 2; Cycs, cytochrome c; MCAD, medium-chain acylcoenzyme A (CoA) dehydrogenase.

(C) Mitochondrial DNA content determined from the ratio of mitochondrial cytochrome $\mathrm{c}$ oxidase 2 (Cox2) to nuclear intron of $\beta$-globin.

(D) Mitochondrial mass determined by Mitotracker green.

All values are means $\pm \operatorname{SE}\left(\mathrm{n}=8\right.$ per group); ${ }^{*} \mathrm{p}<0.05 ; * * \mathrm{p}<0.01 ; * * * \mathrm{p}<0.001$, as assessed by student's $t$ test.

Figure 2. Lipid oxidation in EDL muscle of MPGC-1 $\alpha$ TG mice vs. control animals. (A) Relative expression of genes regulating lipid oxidation was measured by RT-PCR and expressed as fold change over controls. Abbreviations: CPT-1b, carnitine palmitoyl transferase 1b; MCD, malonyl CoA decarboxylase; ACC2, acetyl CoA carboxylase 2.

(B) Palmitate oxidation was assessed ex vivo using tritiated palmitate.

(C) Relative gene expression of citrate synthase as a marker of mitochondrial activity was measured by RT-PCR.

(D) Blood profile of acylcarnitine species was determined using tandem mass spectroscopy and clustered according to saturated (SFA), mono- and polyunsaturated acylcarnitines (MUFA and PUFA respectively).

All values are expressed as means $\pm \operatorname{SE}\left(\mathrm{n}=6-8\right.$ per group); ${ }^{*} \mathrm{p}<0.05 ;{ }^{* *} \mathrm{p}<0.01 ;{ }^{* * *} \mathrm{p}<0.001$.

Figure 3. Oxidative phosphorylation in EDL muscle of MPGC-1 $\alpha$ TG mice vs. control animals.

(A) Relative expression of genes involved in oxidative phosphorylation and uncoupling was measured by RT-PCR and expressed as fold change over controls. Complex I (Ndufb5, NADH dehydrogenase (ubiquinone) $1 \beta$ subcomplex 5); Complex II (SDHB, succinate dehydrogenase complex II, subunit B); Complex III (UQCRC, ubiquinol-cytochrome c reductase complex core); Complex IV (Cox 5b, cytochrome c oxidase, subunit $\mathrm{Vb}$ ); Complex $\mathrm{V}$ (Atp50, ATP synthase, H+ transporting, mitochondrial F1 complex, O subunit); UCP3, uncoupling protein 3.

(B) Membrane potential was assessed in mouse myotubes in vitro.

(C) Relative gene expression for genes involved in $\mathrm{H}_{2} \mathrm{O}_{2}$ production and detoxification; $\mathrm{SOD}$, superoxide dismutase; CAT, catalase; GPX, glutathione peroxidase; GR, glutathione reductase.

(D) Measurement of $\mathrm{H}_{2} \mathrm{O}_{2}$ in EDL muscle of MPGC-1 $\alpha$ TG mice vs. control animals. 
Figure 4. Whole body glucose homeostasis and skeletal muscle insulin sensitivity in MPGC-1 $\alpha$ TG mice $v s$. control animals.

(A and B) Glucose (A) and Insulin (B) tolerance tests in MPGC-1 $\alpha$ TG mice and control animals.

(C) IRS-1 associated PI3K activity in skeletal muscle.

All values are expressed as means $\pm \operatorname{SE}\left(n=6-8\right.$ per group); ${ }^{*} \mathrm{p}<0.05 ;{ }^{* *} \mathrm{p}<0.01$.

(D) Model integrating the findings of the study. PGC-1 $\alpha$ simultaneously acts on lipid oxidation, Krebs cycle and electron transport chain and thus couples all major oxidative pathways. Concomitantly, PGC-1 $\alpha$ promotes the transcription of positive and negative regulators of lipid oxidation and electron transport chain. Thereby PGC-1 $\alpha$ balances oxidative metabolism. Subsequently, excessive metabolism and the generation of acylcarnitines and ROS are prevented.

Abbreviations: CPT-1b, carnitine palmitoyl transferase 1b; MCD, malonyl CoA decarboxylase; ACC2, acetyl CoA carboxylase 2; I-V, complexes I-V; UCP3, uncoupling protein 3; ROS, reactive oxygen species. 


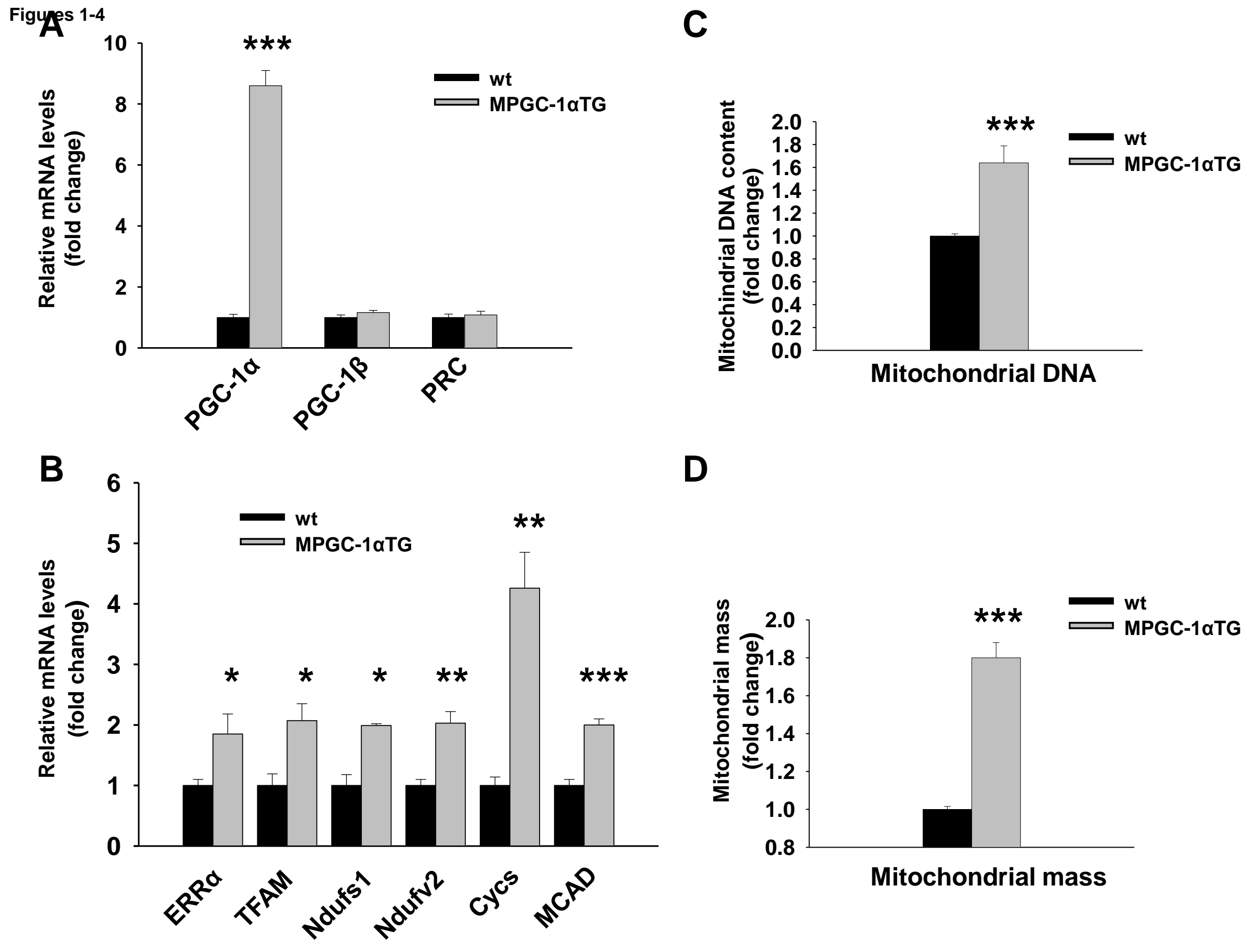

C
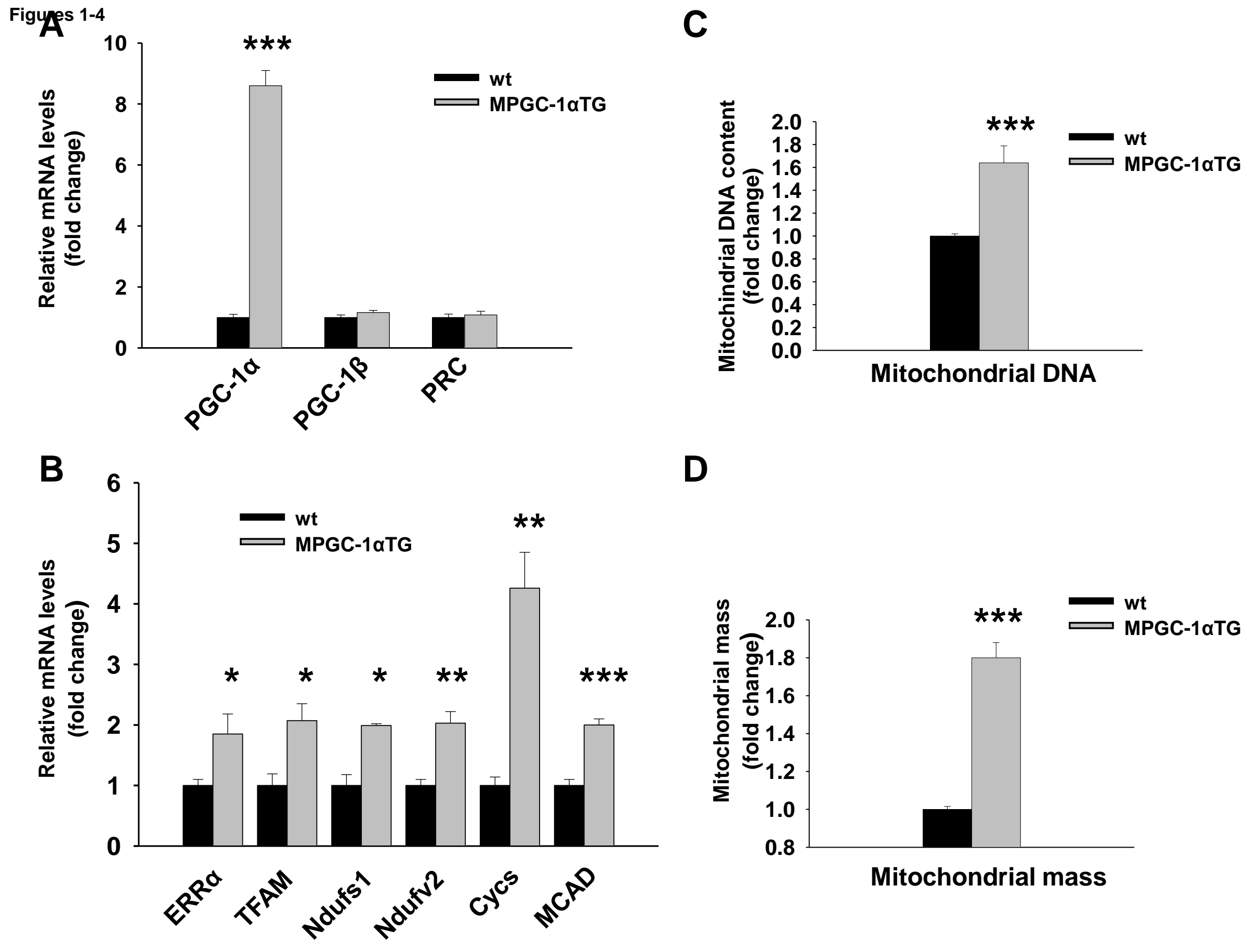

D

Mitochondrial mass 


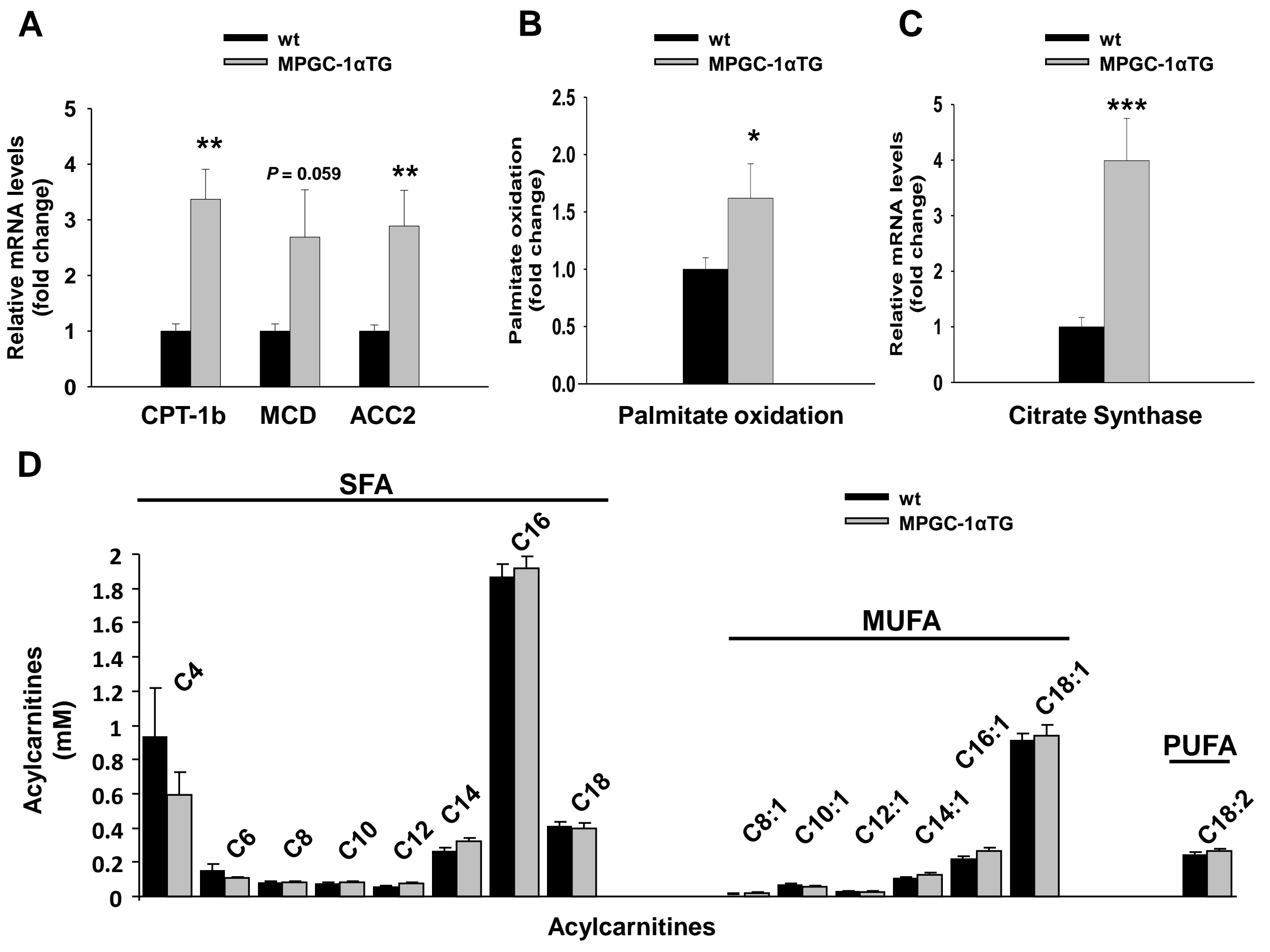




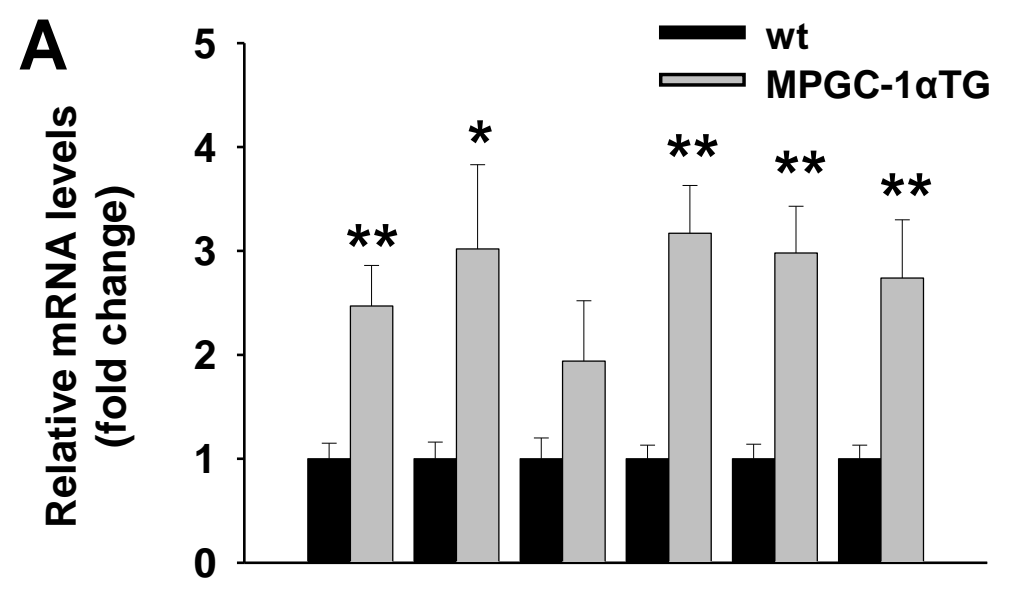

$\begin{array}{lllllll}\text { Complex: I II } & \text { III } & \text { V UCP3 }\end{array}$

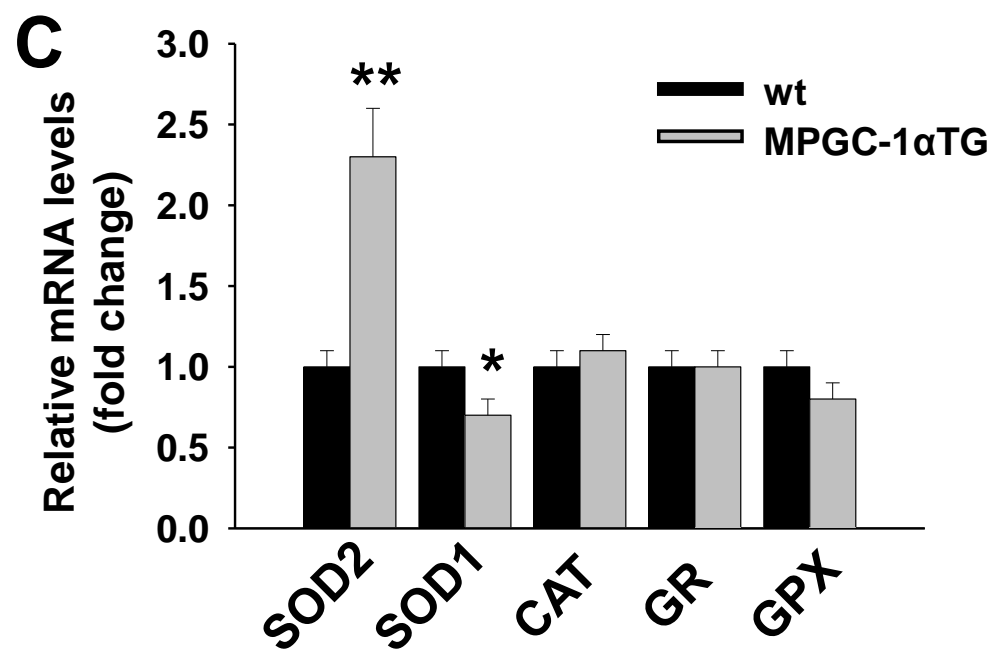

B

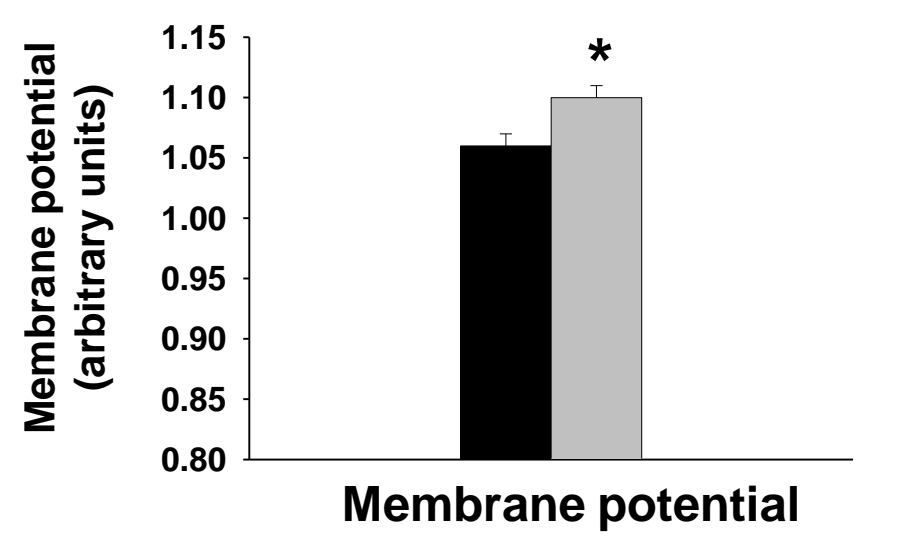

D

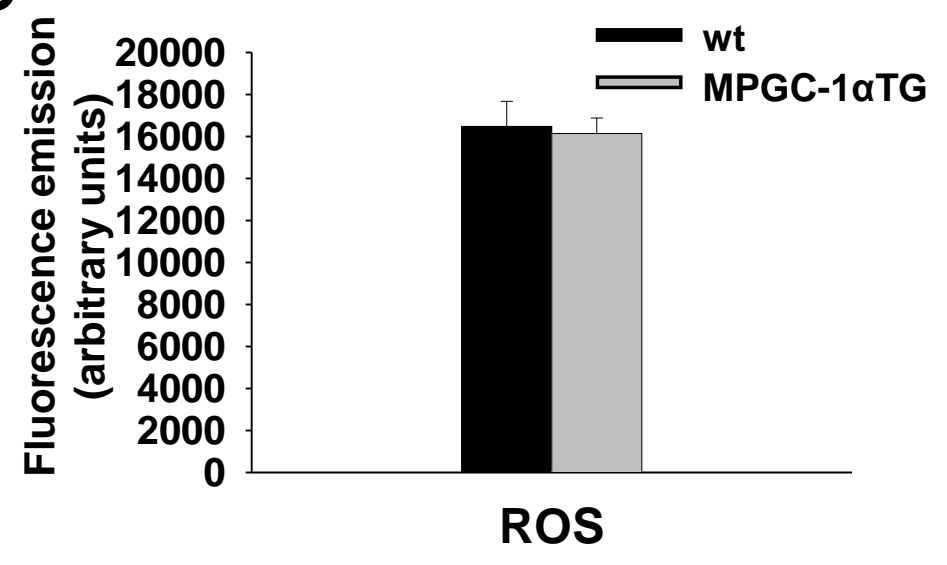


A

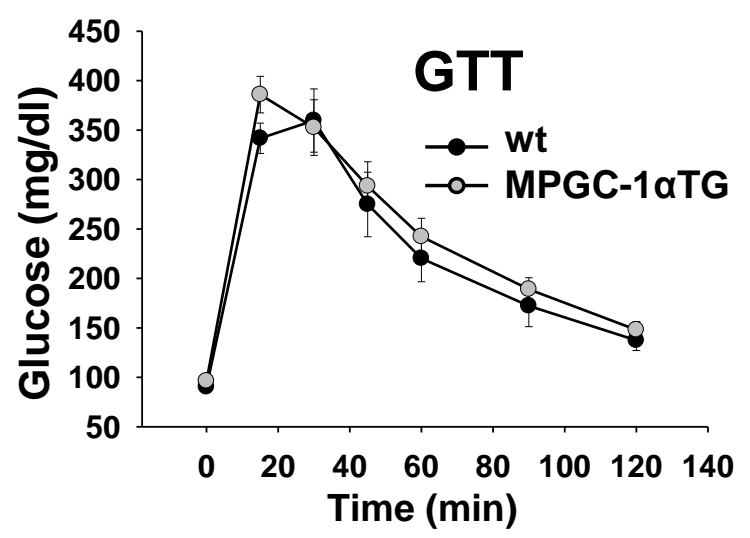

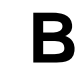

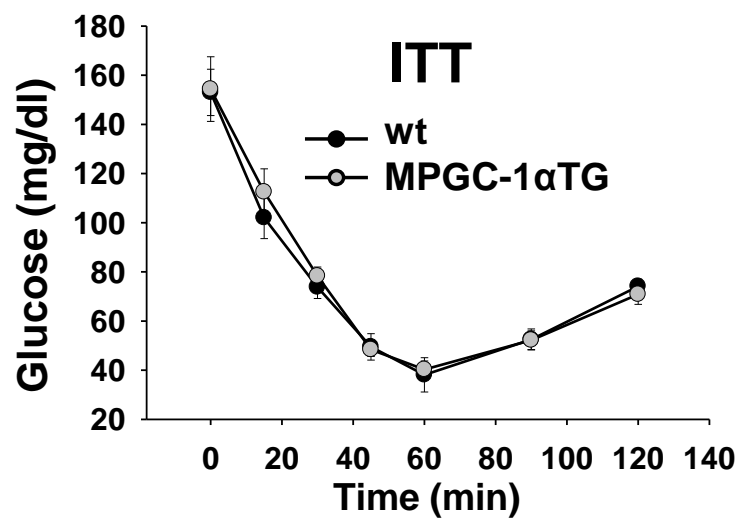

C

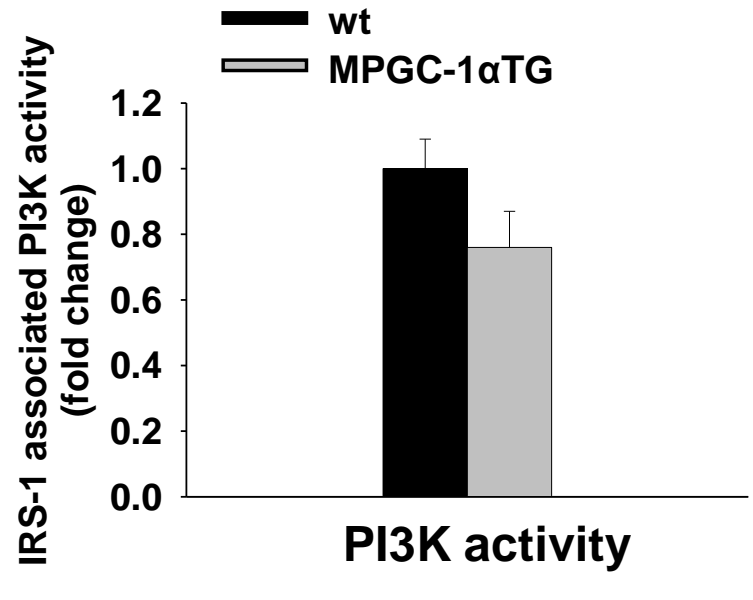

D

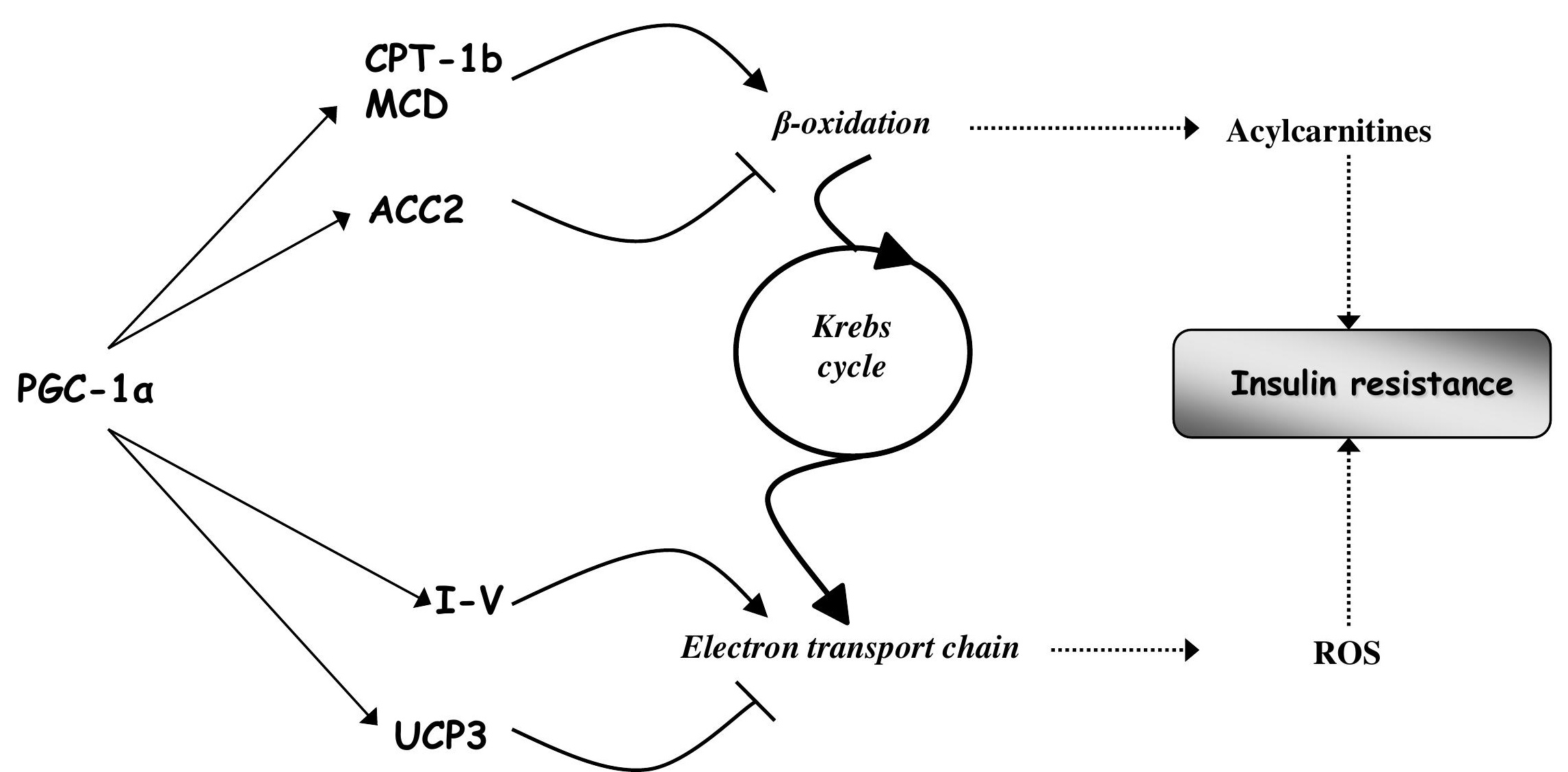

